\title{
RELACIONES SOCIO-AFECTIVAS ENTRE JUGADORES, PADRES, ENTRENADORES Y ÁRBITROS EN LOS CAMPOS DE FÚTBOL BASE
}

Juan Francisco Cara Muñoz', Iván Pernía Fernández² y Manuel Utrilla Abad ${ }^{3}$

'Estudiante $5^{\circ}$ curso de Licenciatura en Ciencias de la actividad física y del deporte. Entrenador de fútbol Nivel II.

${ }^{2}$ Estudiante $5^{\circ}$ curso de Licenciatura en Ciencias de la actividad física y del deporte. Entrenador de fútbol Nivel II.

3Diplomado en Magisterio de Educación Física. Entrenador de fútbol Nacional.

ivan.pernia.fernandez@gmail.com

\section{RESUMEN:}

Este artículo pretende analizar las relaciones que se producen en el fútbol base entre los diferentes elementos o partes, que a la vez influyen en sí mismas y sobre el propio jugador. Desde la perspectiva del entrenador el control de las relaciones que se producen en el terreno de juego, es un aspecto a tener en cuenta dentro del rendimiento formación del deportista, para posteriormente tomar decisiones que favorezcan la formación-educación y el rendimiento del jugador. A su vez, intenta concienciar a las demás partes de la importancia que tienen en el proceso de formación del jugador, para facilitar el trabajo del entrenador y el aprendizaje del jugador.

PALABRAS CLAVE: Fútbol base, relaciones, jugador, padres y formación. 


\section{1.- INTRODUCCIÓN}

Es sabido por todos que en los terrenos de juego, en particular en los de fútbol, nos encontramos distintos tipos de grupos sociales que influyen en los propios jugadores. En el fútbol base se aprecia cómo tanto padres, árbitros, entrenadores como los propios jugadores interactúan de forma que todos conviven interviniendo unos sobre otros.

"El deporte es, quizás, el medio más efectivo de comunicación en el mundo moderno, sobrepasando incluso a las formas verbales y escritas para alcanzar directamente a miles de millones de personas en todo el mundo. No hay dudas que el deporte es una forma viable y legítima de establecer amistad entre las naciones." Nelson Mandela.

Según Kofi Annan, "El deporte puede jugar un rol en el mejoramiento de la vida de los individuos. Y no solamente de los individuos, debería agregar, sino de las comunidades en su conjunto. Estoy convencido que es el tiempo adecuado para edificar ese entendimiento, para alentar a los gobiernos, agencias de desarrollo y comunidades a pensar cómo el deporte puede ser incluido sistemáticamente en los planes de ayuda a la infancia, particularmente de aquellos que viven en medio de la pobreza, la enfermedad y el conflicto".

Para Platón en su libro "La República" (427-347 a.C.) los deportes y la educación física, eran una parte esencial de la educación integral, como la concite hoy el hombre moderno. Ya el filósofo griego definió la educación perfecta así: "La educación es el arte de conducir al niño por los caminos de la razón. Su deber consiste en fortalecer el cuerpo tanto como sea posible y en elevar el alma a su más alto grado de perfeccionamiento."

El deporte según Nogueda (1995), es más que una práctica física, o una forma de canalizar el tiempo libre y los momentos de ocio, es un elemento educativo que constituye un importante fenómeno social.

Gutiérrez Sanmartín (1995), reconoce en el deporte un contexto de alto potencial educativo para la adquisición de valores y desarrollo de actitudes socialmente necesarias.

Este análisis de las relaciones socio-afectivas, ha sido llevado a cabo en el equipo alevín C del Carpetrilla F.C, club perteneciente a la provincia de Sevilla, durante la temporada 2010-2011. La edad de todos los jugadores estaba comprendida entre los 10-11 años, aspecto a tener en cuenta a la hora de analizar dichas relaciones.

A continuación, se expondrán las posibles tipos de relaciones que se pueden dar en los terrenos de juego de fútbol base.

\section{2- RELACIONES SOCIO-AFECTIVAS.}

\section{1.- Relación jugador-entrenador}

La relación que se produce entre el jugador y el entrenador en el fútbol base la podemos diferenciar en dos partes: 


\section{Durante el entrenamiento:}

La relación dentro de un entrenamiento entre el jugador y el entrenador o "míster" como ellos le llaman podemos considerar que es cordial y directa. El entrenador se preocupa de que los jugadores aprendan en un ambiente óptimoagradable en los entrenamientos teniendo siempre presente la seriedad y el trabajo, pero nunca llevándolos al autoritarismo. El míster de estos jugadores cree que es importante tener en cuenta a la hora de preparar las sesiones que son niños de 10 años y que será mucho más productivo trabajar con ellos mediante ejercicios reales y utilizando juegos propios de su edad adaptados al fútbol, que con ejercicios cerrados y en los que no prima la competición. Llegado a este punto resaltar que en estas edades la competición tiene una gran relevancia, ya que los niños se ven motivados cuando en sus actividades está presente dicho factor.

Por otro lado, los jugadores ven al entrenador un sujeto del que pueden aprender el camino para ser mejores jugadores, esto se ve favorecido por el trabajo que el míster realiza con ellos. Es importante que los niños aprecien el esfuerzo y esto solo se consigue trabajando sobre las sesiones e intentando que los juegos no se repitan de forma frecuente, se debe tener en cuenta que los ejercicios se comprenden, se entrenan y por último se automatizan, cuando ya se han automatizado es cuando debemos cambiarlos. Con esto llegar a la reflexión, de que la relación de los jugadores con el entrenador puede ser cordial y seria a la vez, para ellos es importante que desde el primer día se diferencia entre el terreno de juego y el entrenamiento, y el resto del tiempo que pasan en la entidad, a la vez recalcar que es importante dedicarle tiempo a las sesiones, con esto, ellos verán que el entrenador trabaja y las ganas de entrenar de los jugadores aumentarán.

\section{Durante el partido:}

Es frecuente en esta categoría que en el vestuario antes de comenzar el partido encontremos a entrenadores que lanzan mensajes motivadores a sus jugadores para que salgan al cien por cien a jugar, dan consejos de cómo actuar dentro del campo, les explican de forma reducida las estrategias tácticas, los jugadores que lanzan faltas, córner y penaltis. Es mucha la cantidad de información que asimilan en poco tiempo, pero gracias al trabajo de toda la semana, no encuentran problema para realizarlo.

Por otra parte de forma habitual vemos como existen entrenadores que menosprecian el esfuerzo de sus jugadores, ciñéndose sólo al resultado. Es sorprendente como incluso estos jugadores son ridiculizados dentro del campo, en vez de recibir correcciones sensatas de sus entrenadores.

Además de lo citado anteriormente, podemos encontrar situaciones en las que los entrenadores intentan que los jugadores adquieran responsabilidades. En este caso los jugadores eran los encargados de hablar por teléfono con el entrenador en caso de ausencia a un entrenamiento u otro motivo, esto se hacía con la intención de desarrollar en los propios jugadores la competencia en autonomía y habilidad lingüística.

\section{2.- Relación padre-entrenador}

La relación entre padres y entrenador es similar en lo cordial a la anterior, pero por el contrario no siempre es tan directa. En muchas ocasiones son los propios jugadores los intermediarios entre el padre ó madre y el entrenador. Es cierto que 
muchas veces pueden existir malentendidos a la hora de que el niño procese la información y se la transmita a los padres, pero con esto también se consigue que se delegue en el jugador y empiece a asumir responsabilidades. También existen situaciones en las que la relación padre-entrenador es directa en su totalidad. En los terrenos de juego se presencian situaciones, de jugadores en etapa de rebeldía, niños con problemas en los estudios o en sus casas, en las que es imprescindible que el entrenador y el padre o madre del jugador se pongan en contacto y juntos intenten paliar dicho problema. Además de lo citado anteriormente esta relación directa se vuelve a dar cuando un padre se interesa en el progreso de su hijo.

Estas relaciones planteadas anteriormente se caracterizan porque ocurren rutinariamente en las sesiones de entrenamiento. Por otro lado encontramos relaciones que ocurren en el terreno de juego durante el partido, a pesar de ser los mismos individuos los que participan en ellas, cambian notablemente.

\section{3.- Relación entrenador-árbitro}

La relación de los árbitros y los entrenadores dentro de un partido de fútbol base suelen ser oscilatorias.

En los partidos a estas edades, viene a ser frecuente y vergonzoso ver a entrenadores insultar al colegiado y por consiguiente ser expulsados. En esta clase de partidos mayoritariamente debería de primar la formación y educación de los jóvenes deportistas, y sin embargo encontramos individuos que alteran el transcurso normal del partido y el ciclo de aprendizaje de los jugadores.

Por otra parte, encontramos entrenadores que si se compenetran con los árbitros para que los niños puedan crecer deportiva y personalmente. A pesar de existir estas situaciones de cooperación entre árbitro y entrenador, en ligas en las que prima la competición y la victoria es complicado que ocurran. Es importante que el entrenador muestre una actitud de respeto con respecto al árbitro para inculcársela a sus jugadores.

\section{4.- Relación padre-árbitro}

Esta relación es quizás la más complicada dentro del terreno de juego, ya que haga lo que haga el árbitro su decisión va a ser cuestionada por el equipo perjudicado.

Son muchas las ocasiones en las que vemos como padres de los jugadores, menosprecian e insultan a los árbitros encargados de controlar el partido. Estos gritos hacia el colegiado, crea dentro del terreno de juego una tensión que es transmitida a los propios jugadores, a veces provocan que los futbolistas endurezcan su juego y comiencen a aparecer entradas y agresiones fuera de lugar.

De todas estas situaciones se pueden sacar las siguientes conclusiones, con la finalidad de intentar paliar este tipo de actos:

- La primera es que este tipo de personas que van al campo con ánimo de violencia y dar un espectáculo fuera de lo deportivo deberían de desaparecer, ya que da mala imagen al club y no trae nada bueno para la competición.

- La segunda es que en el fútbol base entrenadores, árbitros y padres deberían de actuar en conjunto con el fin de dar ejemplo a sus hijos. Dicen que de tal palo tal 
astilla, si los jóvenes deportistas ven a sus padres o padres de sus compañeros insultando al árbitro, de mayores ellos actuarán de la misma manera.

- Tercero, reflexionar sobre las soluciones que le podríamos dar a situaciones en las que el padre de un jugador actúa de forma errónea, estas soluciones podrían ser las siguientes: hablar con el padre otro día y hacerle ver que ese comportamiento no es bueno para su hijo, amenazarle con que si sigue con esa actitud su hijo jugará menos tiempo ese partido, no citar a su hijo para el siguiente partido e indicarle tanto al padre como al hijo el motivo de la no convocatoria y, por último, que la directiva sancione a dicho padre. Hacer hincapié que en determinados campos es frecuente la venta e ingesta de alcohol por parte de los padres, esto puede provocar que las actuaciones bochornosas antes citadas se vean aumentadas. Ante estas situaciones la competición podría tomar la iniciativa de restar puntos a los equipos en los que se produzcan altercados.

A pesar de presenciar estos actos en los campos de fútbol, resaltar que ocurre en la minoría de las ocasiones, son muchos los padres de jugadores con coherencia y que buscan la formación y educación de sus hijos. Debemos de intentar que los jugadores vean a estos padres como un ejemplo a imitar por todos.

\section{5.- Relación padre-padre}

En general esta relación suele ser buena, son padres que se divierten compartiendo un sábado de fútbol con sus hijos y buscan pasar un rato con ellos realizando una actividad con la que ambos disfrutan, echar un día de fútbol.

A pesar de ello, existen circunstancias en las que esto no se llega a dar, los padres se preocupan más porque sus hijos lleguen a ser futbolistas profesionales que de lo que verdaderamente importa, que es pasar un rato agradable y disfrutar con ellos. En algunas ocasiones, los padres de los futbolistas discrepan de decisiones tomadas por el árbitro o jugadas producidas en el terreno de juego. Tras estas discrepancias es frecuente ver como dichos padres empiezan a insultarse y a faltarse al respeto, insultando incluso a los propios jugadores. Después de analizar dichos acontecimiento en uno de los partidos disputado por el equipo citado en la introducción, se pasó a los catorce jugadores una encuesta sencilla en la que se recogían las siguientes preguntas:

Tabla 1. Encuesta a los jugadores sobre la influencia en el rendimiento de sus padres.

\begin{tabular}{|l|c|c|}
\hline $\begin{array}{l}\text { ¿Crees que los gritos de los padres afectan a tu rendimiento en el } \\
\text { transcurso del partido? }\end{array}$ & SI & NO \\
\hline ¿De qué forma? & MEJORA & EMPEORA \\
\hline $\begin{array}{l}\text { ¿Crees que el árbitro influye en que los padres interrumpan el } \\
\text { partido con gritos e insultos? }\end{array}$ & SI & NO \\
\hline ¿Qué sensación tienes justo antes de que el árbitro de el pitido inicial? (Una palabra) \\
\hline ¿Qué aprendes de estas situaciones vividas en el campo?
\end{tabular}


En la primera y segunda pregunta, ¿Crees que los gritos de los padres afectan a tu rendimiento en el transcurso del partido? ¿Y de qué forma?, nos encontramos como de catorce futbolistas: Uno afirmaba que si le afectaba y que mejoraba, cuatro que si les afectaba y que veían disminuido su rendimiento y por último nueve jugadores a los que les eran indiferentes los gritos de dichos padres. Un dato a tener en cuenta sería el de los cuatro jugadores que empeoran, ya que son doce los jugadores que van convocados a un partido y cuatro sería un tercio. El porcentaje es alto ya que por los gritos de los padres puede ser que el rendimiento del equipo se reduzca notablemente. Es sorprendente como existen niños con diez años que utilizan los gritos e insultos como medio de motivación y concentración en el tiempo que dura un encuentro.

En lo que respecta a la tercera pregunta, ¿Crees que el árbitro influye en que los padres interrumpan el partido con gritos e insultos?, las respuestas se decantan seriamente por que SI influye la actuación del árbitro en el comportamiento de los padres, son solo dos los jugadores que dan la negativa a dicha pregunta. En ocasiones puede ocurrir que las conductas de los padres dentro del terreno de juego se vean influenciadas por las malas actuaciones de los colegiados, ya que en ocasiones existen árbitros que en partidos decisivos no siguen la jugada de cerca, y esto puede dar lugar a errores y fallos que en un partido importante puede costarles la liga a muchos equipos. Por otra parte también destacar que en la mayoría de los casos son los padres que van con la predisposición de amedrentar e insultar al colegiado, y con ello sacar beneficio en el marcador.

En cuanto a la cuarta pregunta, ¿Qué sensación tienes justo antes de que el árbitro dé el pitido inicial?, encontramos diversas sensaciones entre los jugadores del equipo: Nerviosismo, ganas, concentración, responsabilidad, nervios, nerviosismo, ánimo, nervios, nerviosismo, ganas, nervios, intensidad, inquietud y nervios. De todas estas respuestas la predominante es nervios, son más de la mitad los jugadores que antes de iniciar el partido se encuentran nerviosos o con inquietud, este es un factor que se debería trabajar, ya que salir con nerviosismo al campo puede provocar que los primeros minutos se encaje un gol y se comience con una cierta desventaja en el marcador. Una buena opción sería que los jugadores que salen al campo con ánimo y concentración se encarguen de motivar a los que, por el contrario, lo hacen con nervios. Es muy importante afrontar el partido con intensidad para no mostrar debilidad y que el otro equipo se amilane solo por la actitud con la que se juega.

Ante la última pregunta, ¿Qué aprendes de estas situaciones vividas en el campo?, aparecen diferentes respuestas y opiniones:

- Que me tengo que acostumbrar.

- No insultar, ni molestar al árbitro.

- Que hay que estar muy concentrado para no oír lo que se grita fuera del campo.

- Que no hay que insultar a las personas y disfrutar del deporte.

- Que no hay que tener miedo a nada.

- A no hacer caso de lo que pase fuera del campo.

- Que hay que controlar los nervios.

- Que hay que pasar de todo lo que ocurre en el exterior del campo.

- Que con esos comportamientos no se aprende nada, al revés me perjudica.

- Que el árbitro es el que manda.

- Que hay que aguantar lo bueno y lo malo.

- Que no tengo que echar cuenta a los que están fuera del campo.

- Que con las peleas no se llega a ningún sitio. 
- Que no te debes poner más nervios y jugar como tú sabes.

Son diversas las reflexiones de los jugadores y sería convenientes desglosarlas para sacar conclusiones de ellas.

El primer jugador afirma que a estas situaciones se tiene que acostumbrar, ya que en parte si sigue jugando en ligas federadas se va a encontrar continuamente a padres que insultan y gritan, aumentando la tensión y poniendo nervioso a todos los componentes del equipo rival.

El siguiente jugador propone que no insultar ni molestar al árbitro le supondría un beneficio y que por el contrario con palabras malsonantes y recriminaciones no llegará a ningún lado.

El tercero sin embargo, le da más importancia al estar concentrado, ya que con ello conseguirá eludir los gritos e insultos que en el exterior del campo se están produciendo, además de esto, con la concentración logrará salir al campo con un rendimiento óptimo y evitar en los primeros minutos ser sorprendido.

El cuarto jugador generaliza más lo expuesto por el segundo, comenta que no hay que insultar a las personas y disfrutar del deporte, bien es cierto que la finalidad principal de estas ligas es que mediante la competición disfrutemos del deporte aprendiendo sus valores.

Por otro lado, el quinto jugador, expone que no hay que tenerle miedo a nada, es innecesario ponerse nervioso y temerle a los gritos que algunos padres producen con la única finalidad de romper el buen ambiente que una competición deportiva puede tener.

El siguiente, piensa que no se le debe hacer caso a lo que pasa fuera, puede existir una relación entre lo opinado por este futbolista y por el que comentaba que debía estar concentrado para no oír lo que fuera sucede. Esto sería importante tenerlo en cuenta ya que trabajando la concentración se podría evitar que los gritos pusieran nervioso a los jugadores.

El séptimo jugador, comenta que hay que controlar los nervios, gracias a esto podremos estar concentrados y actuar en el partido en función a lo entrenado durante toda la semana. Si entrenamos correctamente durante toda la semana y luego los nervios nos afectan, no servirá de nada tanto esfuerzo.

El siguiente jugador afirma que hay que pasar de todo lo que ocurre en el exterior del campo, la reflexión es similar a la realizada con el sexto futbolista, con la concentración podremos conseguir que los jugadores no se vean perjudicados por los sucesos que ocurren en el exterior del terreno de juego, por ello sería conveniente dedicar parte de los entrenamientos a mejorar la técnica de concentración.

El noveno jugador opina que con estas actuaciones por parte del equipo rival, no aprende nada e incluso puede empeorar su estado. Es cierto y coherente la respuesta, ya que estos jóvenes futbolista están en periodo de aprendizaje y con estos comportamientos los padres están consiguiendo perjudicar la mente de dichos futbolistas y que piensen que estos actos son normales en el mundo del fútbol.

El décimo futbolista afirma que el árbitro es el que manda, directamente relacionado con el que comentó que no se debía de insultar ni molestar al árbitro. Sería conveniente inculcar a los jugadores que el árbitro tiene la potestad y es el que 
decide sobre las reglas del juego, con insultos lo único que se conseguirá es alterarlo e impedir que pueda decidir con coherencia.

El siguiente jugador saca de estos comportamientos que con ellos se aprende a aguantar lo bueno y lo malo del fútbol, este futbolista no deja de lado que existen padres que acuden al campo con el fin de disfrutar del deporte junto con sus hijos, esto sería lo bueno y lo malo es como hemos dicho con anterioridad acuden con el único motivo de romper la dinámica de esta competición.

El duodécimo dice que solo hay que centrarse en el partido y no echar cuenta a los que están fuera. Esta reflexión es similar a la de los dos últimos futbolistas.

EL siguiente futbolista expone que con las peleas no se llega a ningún sitio, y es cierto que porque de la pelea de dos personas se ven perjudicadas otras muchas.

El último futbolista no le da importancia a los gritos y le vuelve a dar importancia a los nervios, afirma que lo importante es no ponerse nervioso y jugar como tú sabes.

\section{6.- Relación entrenador-entrenador}

En cuanto a la relación entre entrenadores antes, durante y después del partido encontramos diferentes cuestiones a tratar y todas ellas posibles dentro de un partido de fútbol base. En primer lugar y lo que viene siendo habitual es encontrarnos a entrenadores que antes de empezar el partido se saludan y desean suerte, durante el partido están metidos en las jugadas y no se dirigen el uno al otro, y al finalizar se dan la enhorabuena y se desean suerte para el resto de la liga. Por otra parte, existen entrenadores los cuales se dedican a intentar desconcentrar al otro equipo mediante palabras malsonantes e incluso gestos antideportivos.

\section{7.- Relación jugador-jugador}

Las relaciones de los jugadores de diferentes equipos dentro del terreno de juego suelen ser respetuosas, antes del partido salen todos juntos al lado del árbitro y se hacen una foto, al terminar el encuentro se dan la mano. A pesar de ser esto lo normal dentro de los setenta minutos que dura el partido de esta categoría, también encontramos jugadores que no respetan estas normas de educación y durante el partido lanzan insultos y amenazas a los jugadores del equipo rival.

Además de las anteriores encontramos relaciones entre los jugadores de un mismo equipo, pueden darse momentos de la liga en los que aparezcan situaciones desagradables entre compañeros. La función del entrenador es intentar reducir las posibilidades de la aparición de dichas situaciones y de crear un buen clima de trabajo. Una de las finalidades es que no sean un grupo sino un equipo, que al final de la liga donde probablemente existan jugadores que tomen caminos distintos se termine con relaciones de amistad.

\section{8.- Relación árbitro-jugador}

Para los jugadores de esta edad el árbitro debe ser un educador más, debe ser flexible y tener la capacidad para enseñar al futbolista que debe y que no se debe 
hacer dentro del campo. En ocasiones nos encontramos a colegiados que tratan a los jugadores de esta edad como si de profesionales se tratase y que piensan que son hombres en miniatura, esta imagen debería de quitarse de la cabeza de muchas personas y con ello lograríamos hacer el aprendizaje de estos futbolistas mucho más fácil.

\section{9.- Relación padre-jugador}

Se ha convertido en una imagen frecuente dentro de los partidos, encontrar a los famosos "padres-entrenadores". Estos padres se caracterizan por dedicarse durante todo el encuentro a cuestionar las decisiones del entrenador de su hijo, y a pronunciar frases tales como "Como puede sacar a ese, si es malísimo". Esta situación llega a su límite cuando estos padres empiezan a insultar a los propios compañeros de su hijo, menospreciando su trabajo.

\section{3- CONCLUSIONES}

No se debería cerrar lo tratado anteriormente sin antes exponer una serie de conclusiones, además de existir unas normas de educación y propias del juego, encontramos personas que las incumplen de forma continuada. Sería más fácil conseguir que todo se cumpliera si existiera una cooperación directa y firme de padres, árbitro, entrenador y los propios jugadores. Es muy importante que todos actúen de forma acorde para conseguir hacer del fútbol un deporte limpio y con el que los niños puedan aprender valores y educación.

Figura 1. Triángulo de relaciones socio-afectivas en el fútbol base.

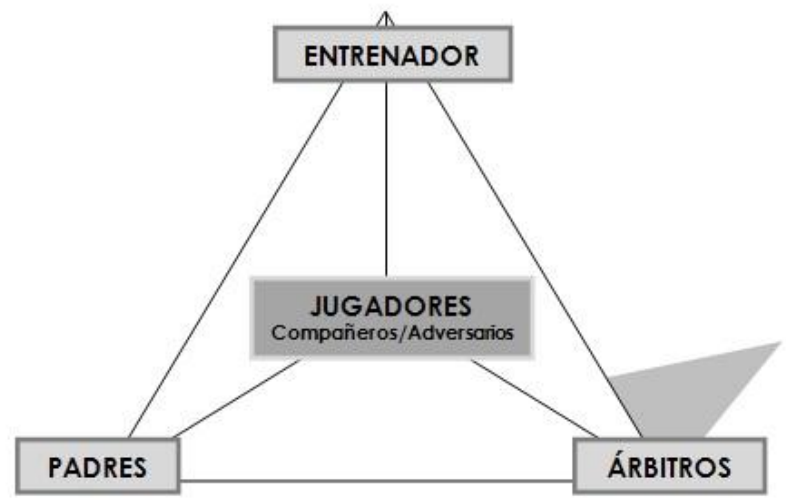

\section{5.- BIBLIOGRAFÍA}

Amigó, E \& Barangé, J. (2004). Adolescencia y deporte. Barcelona. INDE.

Blázquez, D. (1986). Iniciación a los deportes de equipo. Barcelona: Martínez Roca.

Blázquez, D. (1995). La iniciación deportiva y el deporte escolar. Barcelona: INDE Publicaciones.

Carranza, M \& Mora, J.M. (2003). Educación física y valores: educando en un mundo complejo. Barcelona: GRAO.

Castejón, (1995). Fundamentos de la iniciación deportiva y las actividades físicas 
organizadas. Madrid: Dykinson.

Castejón, F. (2003). Iniciación deportiva. La enseñanza y el aprendizaje comprensivo en el deporte. Sevilla: Wanceulen.

Gutiérrez Sanmartín, M (1995). Valores sociales y deporte. Madrid: Gymnos.

Gutiérrez Sanmartín, M. (2003): Manual sobre valores en la educación física y el deporte. Barcelona: Paidós.

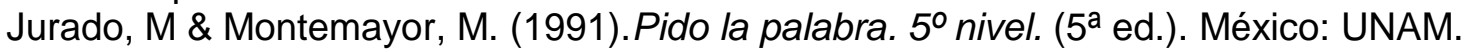
Rodríguez López, J. (2000). Historia del deporte. Barcelona: INDE.

Wein, H. (2000). Fútbol a la medida del adolescente: programas formativos para desarrollar y mejorar su capacidad de juego. Sevilla: Wanceulen.

Wein, H. (2004). Fútbol a la medida del niño (vol.1).Madrid: Editorial Gymnos. 